\title{
The use of autoregression model for maintaining the national time scale of Ukraine
}

\author{
Pavel Neyezhmakov ${ }^{1, "}$, Eduard Koretskyi ${ }^{1}$ \\ ${ }^{1}$ National Scientific Centre “Institute of Metrology”, Myronosytska Str. 42, 61002 Kharkiv, Ukraine.
}

\begin{abstract}
The report presents the results of maintaining the national time scale of Ukraine, UTC (UA), using the prediction recursive lattice filters that indicate high effectiveness results of the proposed algorithms for internal comparisons. Analysis of the data shows that the use of lattice filters allows to keep the national time scale within $\pm 10 \mathrm{~ns}$, that is comparable with the time scales of countries, which have about ten and more hydrogen and cesium standards as well as cesium fountains and generators on cold strontium atoms as part of their primary measurement standards of time and frequency. In the future, the results will be used by the Service of Universal Time and Reference Frequencies of Ukraine.
\end{abstract}

\section{The statement of the problem}

One of the functions laid upon the Head Center of the Service of Universal Time and Reference Frequencies of Ukraine, which is the structural department of the National Scientific Centre "Institute of Metrology", is maintaining the national scale of coordinated time UTC(UA). The Center has been successfully fulfilling this task. Over the past few years, the maximum deviation of the scale UTC (UA) from UTC did not exceed $70 \mathrm{~ns}$ [1]. But the indicators obtained were provided by almost daily analysis of the measurement results of internal and external comparisons of the measurement standard, the introduction, if necessary, amendments and trends as well as calculations and introduction of weighting coefficients for each standard. It should be noted that the modern phase comparators and synthesizer of the physical time scale are absent in the composition of the measurement standard of Ukraine, thus all the calculations and monitoring of the scale are carried out on the daily measurement points. But it is planned to introduce the abovementioned equipment into the composition of the national measurement standard in the nearest future, and after that the generation of the national scale should be conducted in the automatic mode. Such a mode can be provided with the use of more advanced algorithms for processing the measurement results of the system of internal and external comparisons of the national measurement standard of the units of time and frequency of Ukraine.

\section{The analysis of recent researches and publications}

The accuracy of maintaining the time scale is directly dependent on the accuracy of prediction of the scale for a certain period of time [2].
One of the most simple conventional prediction models can be represented in the following form

$$
\hat{x}(t+\tau)=x_{k}(t)+\left[y_{k}(t)+\frac{D_{k} \tau}{2}\right] \tau+\ldots
$$

where $\hat{x}(t+\tau)$ is the prediction of time difference of the scale of $\mathrm{k}^{\text {th }}$ clock in relation to the group scale for the time $\mathrm{t}+\tau$;

$\mathrm{x}_{\mathrm{k}}(\mathrm{t})$ is known time difference of $\mathrm{k}^{\text {th }}$ clock (standard) in relation to the group scale at time $t$;

$\mathrm{y}_{\mathrm{k}}(\mathrm{t})$ is the relative difference in the frequency of $\mathrm{k}^{\mathrm{th}}$ clock in relation to the group frequency;

$D_{k}$ is the constant that determines the linear systematic frequency variation of $\mathrm{k}^{\text {th }}$ clock (standard) in relation to the group frequency.

It is obvious that the predicted value of the scale, which has been calculated at time $t$ for the time $t+\tau$, does not necessarily equal to the value of the scale for the time $t+\tau$. But with this algorithm, the prediction for $t+\tau$ can be corrected using time differences that have been measured at time $t+\tau$ using the equation

$$
x_{k}(t+\tau)=\sum_{j=1}^{N} \omega_{i}\left[\hat{x}(t+\tau)-x_{j k}(t+\tau)\right]
$$

where $\omega_{i}$ is the weighting coefficient of $i^{\text {th }}$ standard in the group, which is determined by the statistical characteristics of the standard.

It follows from the equation (2) that the quality of maintenance of the time scale, primarily, is determined by the ability to predict the behavior of the scale over time. It can be hour, day and other time intervals. Prediction is based on the behavior of individual time

\footnotetext{
* Corresponding author: pavel.neyezhmakov@metrology.kharkov.ua
}

(C) The Authors, published by EDP Sciences. This is an open access article distributed under the terms of the Creative Commons Attribution License 4.0 (http://creativecommons.org/licenses/by/4.0/). 
and frequency standards that are the part of the group. Behavior is determined by the correlation dependencies of the frequency and the scale of the measurement standards at a certain prehistory interval. According to the conventional approach, cesium frequency standards do not have a systematic frequency variation, and hydrogen standards have a linear systematic variation. This particular approach is now used to determine the prediction of scales or frequencies of the standards in relation to the group. In addition, weight coefficients of standards are inserted by the scientist-keeper of the measurement standard in a manual mode and are determined as inversely proportional to the value of the prediction error of a particular standard at a certain time interval.

The analysis of internal and external comparisons of the standards of the measurement standard during several years shows that the behavior of standards is subject to rather complicated laws and is described by polynomials of higher order. In addition, it has been established that the statistical characteristics of the standards are not stationary and may change over time. This is due to the aging of the equipment, the influence of external factors, random frequency variations of the standards etc. From all this it can be concluded that the behavior of the particular standards is a non-stationary correlated random process, for the prediction of which it is required to apply adaptive processing algorithms. Behavior is determined by the correlation dependencies of frequency and scale of the standards on a certain prehistory interval. Therefore, when developing effective algorithms for maintaining the time scale of the standard, it was decided to use already well-known autoregression model, with which it has long been possible to solve tasks of prediction of time series.

In this regard, it is appropriate to use an autoregression model for the maintenance of the time scale, at the basis of which prediction adaptive autoregressive (AR) or lattice filters (LF) can be developed [3].

The autoregression model of random signals has several advantages over other models that makes it widely used for solving a variety of tasks. The main advantages of this model include its constructability, which makes it possible to synthesize schemes and algorithms that allow to generate random processes with given properties. Parameters of AR model of random signals are AR coefficients and the model order. In addition, the model parameters coincide with the coefficients of recursive digital filters. This property greatly simplifies the process of synthesis of this type of filters by a given amplitude-frequency characteristic.

AR model is based on the existence of correlation dependence of the sample of random signal in the current moment of time with some finite number of samples at previous moments of time. Correlation is expressed by the presence of regression of the current sample of the random process on the previous ones. This type of regression is called autoregression. The AR equation can be written in the following form

$$
X_{t}=\sum_{j=1}^{p} A_{j} X_{t-j}+n_{t}
$$

where $A_{j}$ are AR coefficients;

$\mathrm{n}_{\mathrm{t}}$ are uncorrelated random samples of white noise type; $\mathrm{p}$ is the order of AR model.

The value

$$
\widetilde{X}_{t}=\sum_{j=1}^{p} A_{j} X_{t-j}
$$

is called the prediction of a random value. The difference between the current value of the sample and its prediction is called the prediction error

$$
n_{t}=X_{t}-\tilde{X}_{t}
$$

Building of AR model of a random process is reduced to finding AR coefficients and $\mathrm{p}$ order. The Yule-Walker equations allow to determine the unknown AR coefficients $\mathrm{A}_{\mathrm{i}}$.

The development of the theory of prediction of time series led to the synthesis of so-called lattice filters. Building of AR model or the synthesis of AR filters requires calculating the AR coefficients. To do this, it is needed to rotate the correlation matrix, and this operation is associated with a large amount of calculations. The search for effective algorithms of calculating AR coefficients led to the synthesis of lattice structures. LF parameters are the reflection coefficients and the number of filter sections. The number of sections is equal to the order of AR model. Like AR filters, LF ones are also prediction filters that minimize the dispersion of prediction error. The values of reflection coefficients are mutually simultaneously related to AR coefficients and finally determined by the correlation function. The transformations that link a set of reflectance coefficients to AR coefficients are nonlinear ones. For realization of specific prediction filter by means of software or hardware, both direct implementation and lattice structure can be used. Both realizations are mathematically equivalent, but there are a number of practical differences between them that make the lattice structure a preferable option.

When building AR models, either block or adaptive processing of input data is used. Block processing is that the array of input data of a certain size is memorized, then it is processed and AR model is built. Block processing makes sense in the case when the random process is stationary, that is, the form of autocorrelation function does not change over time. But in our case, we deal with non-stationary random processes, as the correlation properties of time and frequency standards that are part of the standard change over time. First of all, this is manifested in random frequency variations of the standards. Therefore, it is necessary to use adaptive data processing, which is that the coefficients of the prediction model need to be updated as often as possible so that they would be in time with changes of the correlation properties of the input processes. 
An adaptive algorithm is developed for whitening LF, which updates its reflection coefficients with every new input sample of random process [3]. In the scientific literature, it is called the recursive least squares lattice algorithm. The performed additional comparative studies indicate that the lattice recursive structure has the highest convergence rate compared to other similar adaptive algorithms [4].

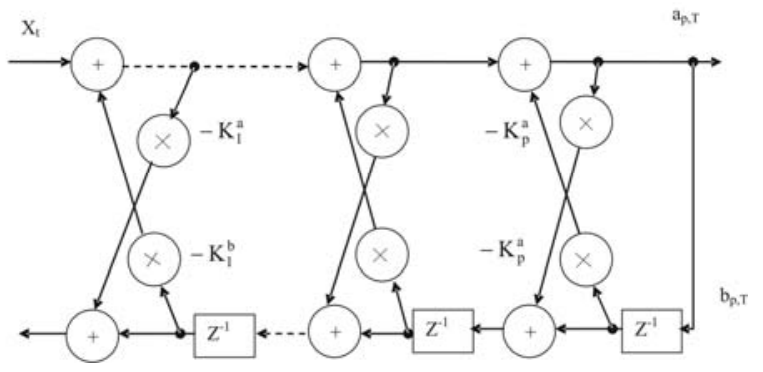

Fig. 1. Synthesizing lattice filter

Figure 1 shows a block diagram of a synthesizing lattice filter or a prediction filter.

\section{The results of experimental studies}

When maintaining a national time scale, it is necessary to ensure that two conflicting requirements are met. On the one hand, the maximum accuracy, that is, the minimum deviation from UTC scale. On the other hand, the ability to independently maintain the scale for a certain period of time, without falling outside the set deviations from UTC scale. By autonomy we mean the absence of any external comparisons. In the autonomous operation mode, for the maximum deviations of the scale, the requirements specified in the certificate of the national primary measurement standard of Ukraine can be accepted, namely, the differences between the national coordinated time scale of Ukraine UTC (UA) and the international coordinated time scale UTC should be within $\pm 1 \mu \mathrm{s}$.

Figure 2 shows the national time scale UTC (UA), which was obtained using a recursive lattice prediction algorithm. The scale was built on the time interval of more than two years on the basis of daily averaging points. It should be emphasized that the scale was built in a fully automatic operation mode. The maximum deviation from UTC scale was 75 ns. It became possible to achieve such a result only at the expense of more effective prediction algorithm. At the same time, the weighting factors of the standards of the group were unchanged, and corrections did not apply to the trends of changing the group frequency of the measurement standard and individual standards in the group.

At this stage, studies were performed on the effectiveness of applying autoregression prediction algorithms in comparison with conventional algorithms based on assumptions that hydrogen standards have a linear systematic frequency variation, that is absent in cesium standards. The main criterion for efficiency evaluation can be the root-mean-square (RMS) deviation of a prediction error for each standard in the group in relation to the group frequency. In application of autoregression algorithms, the RMS deviation for three hydrogen standards was $1.7 \cdot 10^{-14}, 1.9 \cdot 10^{-14}, 2.2 \cdot 10^{-14}$ relative units and $3.9 \cdot 10^{-14}$ for cesium standard. When applying the conventional prediction algorithms, these values were almost 2 times worse for hydrogen standards and 3 times worse for cesium one. At the same time, also the issues related to determining the optimal orders of prediction filters were studied. For determining the best orders, there are many criteria that are described in detail in the literature, for example in [4]. But in our case, the most simple but effective criterion was used, which calculates the minimum of prediction error depending on the order of the filter. It was determined that for prediction of the behavior of hydrogen standards, the $3^{\text {rd }}$ order of the recursive lattice filter is the best, and for the cesium ones, the $2^{\text {nd }}$ order of the daily averaging points is the best. The third order of the prediction filter for hydrogen standards shows that the behavior of these standards corresponds to more complicated laws than is assumed by the conventional theory.

At the second stage, the issues of development of more efficient algorithms for calculating the trends of group frequency of the standard were studied. They arise due to the fact that sometimes there are situations when the frequencies of several standards in the group at the same time immediately change in one direction. It is naturally that the group frequency of the standard also changes in the same direction, but to find out such a situation by the analysis of the results of internal comparisons is quite difficult and, in most cases, is completely impossible. In this case, it is necessary to use the results of external comparisons, on the basis of which the group frequency trend is calculated and the trend corrections are introduced. The process of trend determining also includes the prediction problem, so for the calculation and introduction of corrections, a lattice filter at the daily points has also been used.

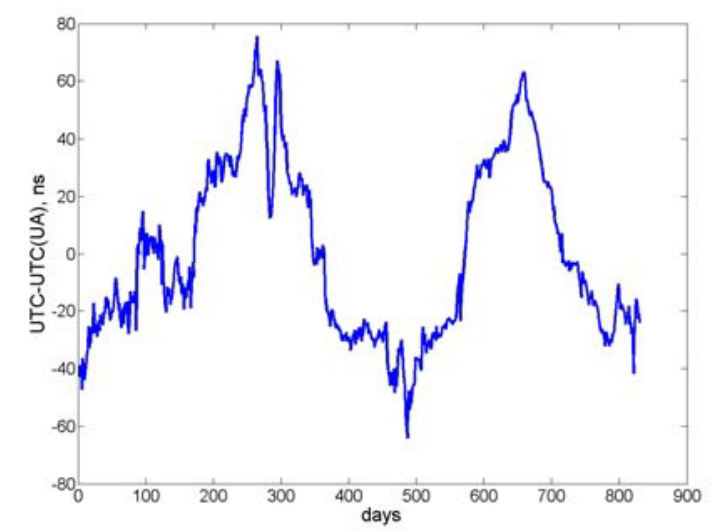

Fig. 2. UTC (UA) scale in relation to UTC scale

Figure 3 shows the difference between UTC (UA) and UTC scales in the same time interval with the automatic deletion of the group frequency trend of the measurement standard every day with a lattice recursive prediction filter. Analysis of Fig. 3 indicates that, in this 
case, the maximum deviation of UTC (UA) scale from UTC scale does not exceed 10 ns.

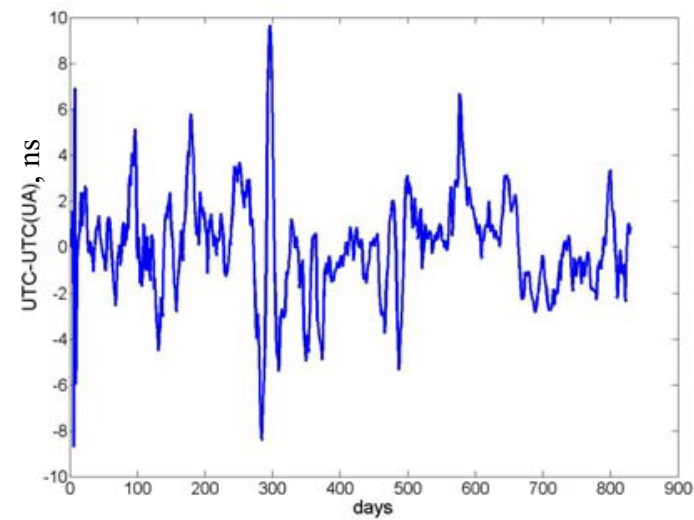

Fig. 3. UTC (UA) scale in relation to UTC scale after the introduction of corrections for the group frequency trend

\section{Conclusion}

Thus, the results of experimental researches indicate that the use of lattice recursive prediction filters for maintenance of the national scale UTC (UA) is effective and allows to ensure the accuracy of maintenance of the scale within $\pm 10 \mathrm{~ns}$ in relation to UTC scale. In addition, due to the adaptive properties of the proposed algorithm, it is possible to independently maintain the national scale for more than two years with a maximum deviation from UTC that does not exceed $100 \mathrm{~ns}$. The proposed and investigated algorithms can be effectively used for the national primary measurement standards for the maintenance of the national time scales.

\section{References}

1. P. Neyezhmakov, A. Prokopov, V. Romanko, The Time and Frequency Measurement Standards of Ukraine // 16-th Int. Congress of Metrology, 17002 (2013)

2. J. M. Lopez-Romero, N. Diaz-Munoz,

M. A. Lombardi, Simp. of metrology, (2008), 3441

3. P.M. Grant, C. F. Cowan at all. Adaptive filters: Transl. from Eng. (M., Mir, 1990).

4. B. Friedlander, TIIER, (1982), 8, 70. 